"This is an Accepted Manuscript of an article published in International Journal of Polymeric Materials and Polymeric Biomaterials on 13 Jan 2015, available online: http://www.tandfonline.com/10.1080/00914037.2014.977900."

\title{
Dose-dependent effects of gamma irradiation on the materials properties and cell proliferation of electrospun polycaprolactone tissue engineering scaffolds
}

\author{
Robin Augustine, ${ }^{\text {a }}$ Abhijit Saha, ${ }^{\mathrm{b}}$ V.P. Jayachandran, ${ }^{\text {c\# }}$ \\ Sabu Thomas, ${ }^{\mathrm{a}, \mathrm{d}^{*}}$ Nandakumar Kalarikkal ${ }^{\mathrm{a}, \mathrm{e}^{*}}$
}

\begin{abstract}
${ }^{a}$ International and Inter University Centre for Nanoscience and Nanotechnology, Mahatma Gandhi University, Kottayam, Kerala 686560, India.

${ }^{\mathrm{b}}$ UGC-DAE Consortium for Scientific Research, Kolkata 700098, India.

${ }^{c}$ Antibacterials and Microbial Technology Lab, Pushpagiri Research Centre, Pushpagiri Institute of Medical Sciences, Tiruvalla, Kerala 689 101, India.

\# Present address: Department of Food Science, University of Pretoria, Hatfield 0028, Pretoria, South Africa

${ }^{\mathrm{d}}$ School of Chemical Sciences, Mahatma Gandhi University, Kottayam, Kerala 686560, India.

${ }^{\mathrm{e}}$ School of Pure and Applied Physics, Mahatma Gandhi University, Kottayam, Kerala 686560, India.
\end{abstract}

\footnotetext{
*Corresponding authors: Sabu Thomas (Email: sabupolymer@yahoo.com, Fax: 91-481-2590357); Nandakumar Kalarikkal (nkkalarikkal@mgu.ac.in)
}

\begin{abstract}
Electrospun membranes of polycaprolactone are widely used for biomedical applications like wound dressings and tissue engineering scaffolds. It is important to sterilize this material using the most accepted method, the gamma irradiation. In this study, we have evaluated the sterilizability of electrospun polycaprolactone membranes with gamma radiation of varying doses. The irradiated materials were assessed for the changes in morphology, crystallinity, surface degradation, hydrophilicity, mechanical property, sterility and the cell proliferation. Our results demonstrate that electrospun polycaprolactone can be effectively sterilized by gamma irradiation, however a higher dose of radiation affect the
\end{abstract}


materials properties. The irradiated membranes showed improved hydrophilicity and fibroblast cell proliferation.

Key words: Polycaprolactone; electrospinning; gamma radiation; sterilization; cell proliferation

\section{Introduction}

Polycaprolactone (PCL) is a biocompatible and biodegradable polymer with immense potential as a biomaterial for various biomedical applications including tissue engineering scaffolds, wound dressings, skin substitutes etc. [1-5]. Electrospinning of PCL has been reported by many workers for the past few decades $[1,2,6,7]$.

Gamma irradiation is the best method of sterilization, because it kills bacteria by breaking down bacterial DNA, thereby inhibiting bacterial division [8]. Energy of gamma rays passes through the material, disrupting the pathogens that cause contamination. Such photon-induced damage at the molecular level leads to the death of microorganisms. Earlier, a minimum dose of $25 \mathrm{kGy}$ was routinely applied for the sterilization of many medical devices, pharmaceutical products and biological tissues. Presently, as recommended by the International Organization for Standardization (ISO), the sterilization dose must be set for each type of product depending on its characteristics and the load of microbes. In addition to killing bacterial cells, radiation may also affect physical and chemical properties of the polymers [9]. Gamma irradiation has also been utilized to modify the porous structure of tissue engineering scaffolds [10]. Irradiation causes the photons to penetrate the material, breaking the polymer chains and creating free radicals. These free radicals can also recombine to create crosslinks between adjacent molecules [11].

Before using radiation for the sterilization of biomedical devices, it is necessary to find out whether the radiation may have any effect on the materials used in the devices [12]. These changes in polymers depend on the radiation doses, internal structures of polymers such as types of functional groups present, chain lengths, etc. [13]. The primary changes can be chain scission and/or crosslinking [14]. Material degradation leads to the loss of mechanical properties, while crosslinking results in the initial increase in tensile strength. In general, aliphatic polymers are susceptible to high-energy radiation [15]. Since the PCL is an aliphatic polymer, gamma radiation may have some influence on the physical properties of these materials. Thus, it is important to optimize the dosage of gamma irradiation required to 
successfully sterilize the PCL membranes as well as to study the physico-chemical changes induced by gamma photons in the polymeric membrane.

\section{Materials and methods}

\subsection{Materials used}

Polycaprolactone (Mw 70,000) used in this study was obtained from Sigma Aldrich, St. Luis, USA. Acetone was obtained from Merck, Mumbai, India. Dulbecco's modified eagles medium (DMEM), fetal bovine serum (FBS), trypsin/EDTA (Ethylenediaminetetraacetic acid) solution, Fluid thioglycollate medium and nutrient agar were purchased from HiMedia laboratories, Mumbai, India. All the reagents used in this study were of analytical grade quality and therefore used without further purification.

\subsection{Fabrication of electrospun PCL membranes}

PCL membranes were prepared by electrospinning of PCL in acetone. The electrospinning apparatus was assembled by Holmarc, India and consisted of a syringe pump and a high voltage power supply. The tip to collector distance was maintained at $15 \mathrm{~cm}$ with an applied voltage of $18 \mathrm{kV}$. The flow rate was maintained as $1 \mathrm{ml} / \mathrm{h}$. As the collector, a thin aluminum sheet of $7 \mathrm{~cm}^{2}$ was attached to the stationary collector which was grounded properly. A relative humidity of 55\% was maintained throughout the experiment.

PCL solution of 15 wt. v\% was prepared in acetone and stirred in a magnetic stirrer for 12 hours for the dissolution of the pellets. About $10 \mathrm{ml}$ of the prepared solution was taken in plastic syringe and electrospun on aluminum sheets.

\subsection{Exposure to Gamma irradiation}

Electrospun PCL membranes were irradiated with ${ }_{60} \mathrm{Co} \gamma$-source that is capable of emitting photons with an average energy of $1.25 \mathrm{MeV}$ at dose rate of $4.7 \mathrm{kGy} / \mathrm{h}$ with an accumulated dose of 15, 25, 35 and $65 \mathrm{kGy}$. ${ }_{00} \mathrm{Co}$ gamma irradiation experiment (cylindrical irradiation chamber with length $14 \mathrm{~cm}$ and diameter $10 \mathrm{~cm}$ ) was conducted at UGC-DAE Consortium for Scientific Research, Kolkata Centre.

\subsection{Scanning Electron Microscopy}

The morphological features of the membranes before and after gamma irradiation were determined by scanning electron microscopic (SEM) analysis. The membranes were carefully sectioned with an approximate size of $3 \mathrm{~mm}$ length and $0.5 \mathrm{~mm}$ width and mounted 
onto an SEM sample holder. Prior to the examination, each sample was coated with platinum using a JEOL JFC 1600 Autofine coater. A JEOL JSM 6390 SEM at $30 \mathrm{kV}$ was used to analyze the samples.

\subsection{Fourier Transform Infrared Spectroscopy}

FTIR analysis has been carried out before and after the gamma irradiation study to understand the changes in the surface functional groups during irradiation. FTIR spectra were obtained from a Perkin Elmer, spectrum 400 FTIR spectrometer. The spectra were collected over a range of $400-4000 \mathrm{~cm}^{-1}$.

\subsection{X-Ray Diffraction Analysis}

X-Ray Diffraction (XRD) analysis was carried out in order to understand the changes in the crystallinity and the structure of the PCL membranes after the gamma irradiation. XRD was recorded in the $2 \theta$ range of $15^{\circ}-30^{\circ}$ using a model D8-Advance of Bruker (Germany), of $\mathrm{CuK} \alpha$ radiation, the energy of which was $8.04 \mathrm{keV}$ and wavelength was $1.54 \AA$. The applied voltage was $40 \mathrm{kV}$ and current was $25 \mathrm{~mA}$.

\subsection{Contact angle measurement}

The water contact angle on the surface of the electrospun PCL membranes was measured by a digital contact angle measurement system (Phoenix) equipped with a CCD camera (KGV-5000). The electrospun membranes after gamma irradiation were placed on the stage of the contact angle machine. A small water droplet (Milli-Q-water) was carefully placed on the surface of the electrospun membranes using the software controlled system. A series of images of the solution droplets were automatically taken. Contact angle was measured from these images. Three samples from each irradiation dose and three measurements were carried out for each sample. The results were expressed as means of these nine measurements ( \pm standard deviation).

\subsection{Tensile strength measurements}

The effect of gamma irradiation on the mechanical strength of the polymer membrane has been evaluated using tensile testing. Tinus Olsen H50 KT Universal Testing Machine was used according to ASTM D 882 standard by applying a $500 \mathrm{~N}$ load cell at a crosshead speed of $1 \mathrm{~mm} / \mathrm{min}$. Gamma irradiated and unirradiated samples were cut into rectangles with dimensions of $6 \times 1 \mathrm{~cm}^{2}$ and vertically mounted in between two mechanical gripping units of 
the tester, leaving a $3 \mathrm{~cm}$ gauge length for mechanical loading. The sample thicknesses were measured with a high precision electronic micrometer. The average values of the tensile property were obtained from the results of five tests and expressed as mean \pm S.D.

\subsection{Evaluation of gamma sterilization efficiency (According to ISO 11737-2: 2009 standard)}

After the gamma irradiation, the samples were sealed in sterile polythene covers and transported to a microbiology lab to study the effectiveness of sterilization. $500 \mathrm{mg}$ of samples (unirradiated PCL membranes and the PCL membranes irradiated with different doses of gamma radiation) were transferred to separate sterile $10 \mathrm{ml}$ test tubes containing fluid thioglycollate medium. Then these tubes were plugged with cotton and incubated for 14 days at $37{ }^{\circ} \mathrm{C}$. In order to detect the presence or absence of microbial growth, during incubation, these media containing tubes were checked every day for visible evidence of growth till the $14^{\text {th }}$ day. The possible degradation of PCL rendered the medium turbid so that the presence or absence of microbial growth cannot be readily determined by visual examination or spectrophotometric analysis. Hence, $10 \mu \mathrm{l}$ medium from each tube was streaked on nutrient agar plates and incubated at $37{ }^{\circ} \mathrm{C}$ for 48 hours. Additionally, after 14 days of incubation, $1 \mathrm{ml}$ of medium from each tube was aseptically transferred into $10 \mathrm{ml}$ of fresh thioglycollate medium and further incubated at $37{ }^{\circ} \mathrm{C}$ for 4 days. After 4 days, $10 \mu 1$ medium from these tubes were streaked on separate nutrient agar plates and incubated at 37 ${ }^{0} \mathrm{C}$ for 48 hrs. Absence of colonies in the first set (from the tubes underwent first 14 days incubation) and second set (from the tubes underwent 4 additional days incubation) of nutrient agar plates after incubation confirmed the sterility of the product. 48 hours of incubation of culture plates ensures the absence of even slow growing microorganisms.

\subsection{Fibroblast cell proliferation}

L929 fibroblast cell lines were purchased from NCCS Pune was maintained in Dulbecco's modified eagles medium supplemented with $10 \% \mathrm{FBS}$ and grown to confluency at $37{ }^{\circ} \mathrm{C}$ in $5 \% \mathrm{CO}_{2}$ supply in a humidified atmosphere in a $\mathrm{CO}_{2}$ incubator (NBS, Eppendorf, Germany). The cells were trypsinized (500 $\mu 1$ of $0.025 \%$ Trypsin in PBS/0.5 mM EDTA solution) for 5 minutes and passaged to $\mathrm{T}$ flasks in complete aseptic conditions. Cells were cultured for 24 hours in scaffolds with unirradiated membranes and membranes treated with varying doses of gamma radiation in fibroblast culture medium. The samples were cut into $1 \mathrm{x} 1-\mathrm{cm}$ square shapes and used for cell 
culture. All the membranes irrespective of the irradiation dose were further sterilized with $70 \%$ of alcohol for $20 \mathrm{~min}$ and both sides of the scaffold were exposed to ultraviolet irradiation in a laminar flow hood for 20 min. Prior to cell seeding, the scaffolds were soaked in warm culture medium for 24 hours, dabbed gently on sterile blotting paper to remove excess moisture and placed in a 24 -well plate. Cell pellet was suspended in culture medium and $30 \mu \mathrm{l}$ of cell suspension containing 50,000 cells were pipetted on top of each scaffold.
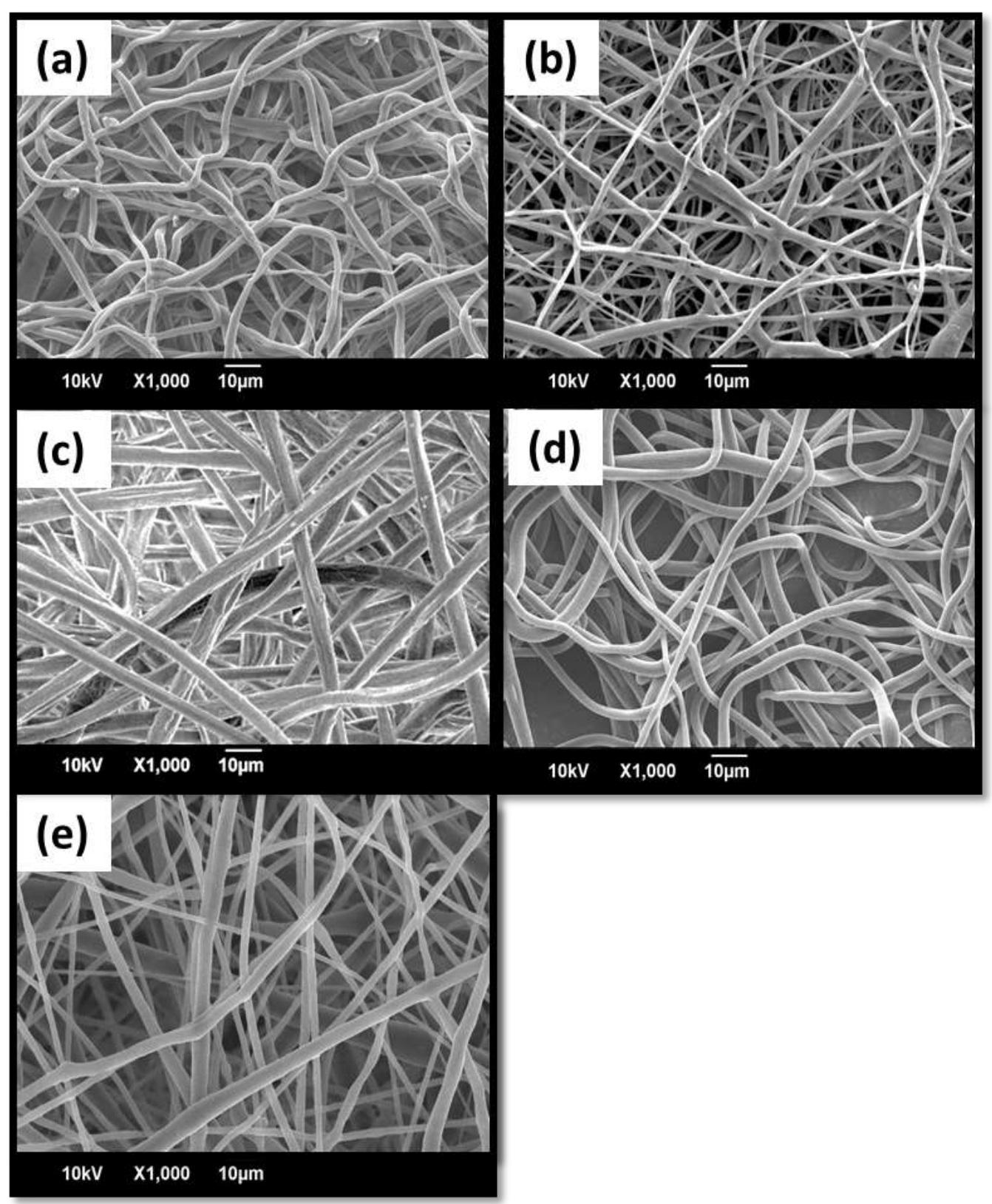

Figure 1: Representative SEM images of electrospun PCL membranes before (a) and after gamma irradiation with $15 \mathrm{kGy}$ (b), $25 \mathrm{kGy}$ (c), $35 \mathrm{kGy}(\mathrm{d})$ and $65 \mathrm{kGy}(\mathrm{e})$ of irradiation dosage. 
The plate was incubated at $37^{\circ} \mathrm{C}$ for $40 \mathrm{~min}$ for cells to attach before $1000 \mu \mathrm{l}$ of culture medium was added into each well. After the addition of medium, plates were incubated in incubator with $5 \% \mathrm{CO}_{2}$ supply at $37{ }^{\circ} \mathrm{C}$ for 24 hours. After 24 hours the culture plates were observed under an inverted phase contrast microscope (No. X51, Olympus, Japan) and images were taken using a CCD camera attached to the microscope.

\section{Results and discussions}

\subsection{Morphology (SEM)}

Scanning electron microscopy (SEM) was used to find out the effect of gamma irradiation on the morphology of electrospun PCL membranes (Figure 1). From the scanning electron micrographs, it is clear that there was no considerable variation in fiber morphology during gamma irradiation. There were no obvious signs of damage across the dose range in the membranes.

\subsection{FTIR}

The radiation induced degradation of electrospun PCL membranes were determined by FTIR analysis. The FTIR spectra of PCL membranes before and after irradiation with varying doses of gamma irradiation were taken (Figure 2). FTIR spectrum before irradiation

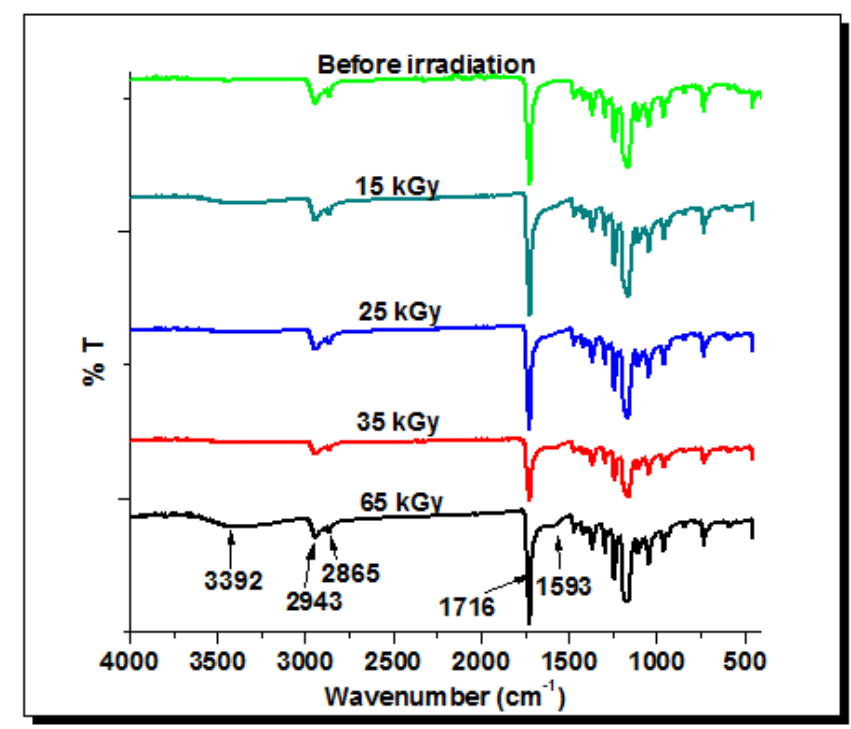

Figure 2: FTIR spectra of electrospun PCL membrane after the exposure to varying dosages of gamma irradiation. 
shows an intense peak at $1716 \mathrm{~cm}^{-1}$ which is due to the presence of the ester carbonyl group that corresponds to the $-\mathrm{CO}$ group in PCL. The peaks at 2865 and $2943 \mathrm{~cm}^{-1}$ are related to the $\mathrm{C}-\mathrm{H}$ bond of saturated carbons. Apart from these characteristic peaks two additional peaks were also observed in the case of gamma irradiated membranes, one broad peak at 3402 to $3440 \mathrm{~cm}^{-1}$ (OH stretching vibrations) and another one at 1586 to $1593 \mathrm{~cm}^{-1}$ (C=O group). These peaks indicate the presence $\mathrm{OH}$ (hydroxyl) functional group and $\mathrm{COOH}$ (carboxyl) group in the PCL membrane after the irradiation due to the cleavage of ester

bonds. Polyesters like PCL have ester bonds on their backbone (main) chains which are liable to cleavage. Gamma irradiation has been known to cause scission of polymer molecular chains, breaking them into smaller fragments [16]. FTIR analysis shows a higher intensity of the $\mathrm{O}-\mathrm{H}$ stretching band as the radiation doses increase. This phenomenon suggests that an increase in dosage of the gamma radiation affects the polymer network. Ionizing radiation is composed of particles that individually carry enough kinetic energy to liberate an electron from an atom or molecule by ionizing it [17]. Gamma radiation can cause damage to the molecular structure of the polymer either as a result of the direct transfer of energy to the molecules or as a result of the secondary electrons released by ionization. Ionizing radiations generate free radicals by the emission of secondary electrons which prolongs peroxidative radical mechanisms. This results in the polymer degradation due to chain cleavage. Further these high-energy treatments result in free radicals generation, main chains scission and crosslinking, and subsequently alter the distribution of chains size in the bulk polymer. Interand intra-molecular rotations and translations may take place when the macromolecules of the polymer mixtures absorb gamma radiations.

\subsection{Water contact angle}

The surface hydrophobicity of a material can be assessed by measuring the contact angle through water spread of a droplet on the surface. If the contact angle is less, the surface of the sample will be more hydrophilic. Result of water contact angle measurements of the electrospun PCL membranes before and after the gamma irradiation obtained by the sessile drop technique is given in Figure 3 and Table 1. To determine the effect of gamma irradiation in the hydrophilicity of electrospun PCL membranes, the contact angle between each membrane after irradiating with varying doses viz. $15 \mathrm{kGy}, 25 \mathrm{kGy}, 35 \mathrm{kGy}$ and $65 \mathrm{kGy}$ were measured. The contact angle of the unirradiated membranes was much higher than the irradiated membranes. From Table 1, it is evident that the initial contact angle of the 


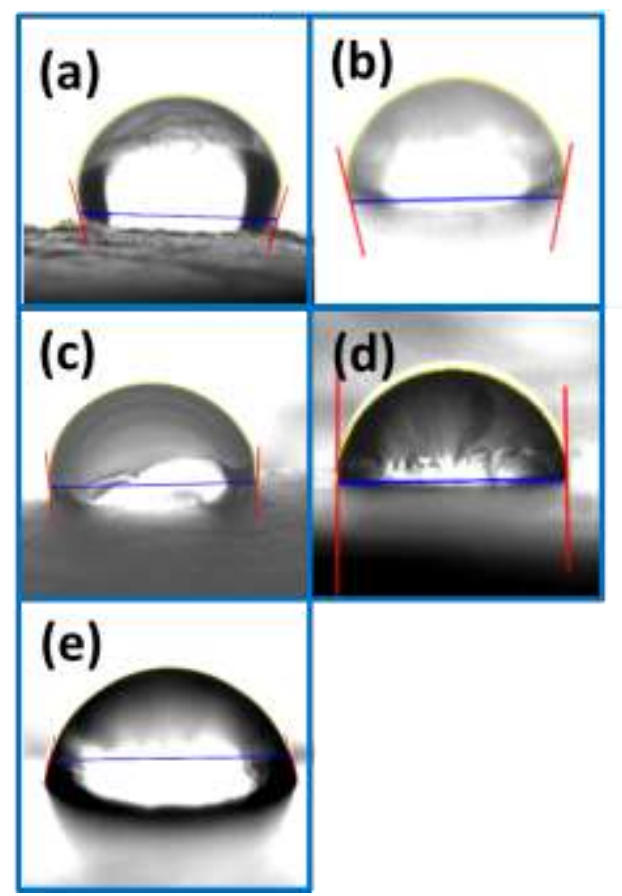

Figure 3: Snapshots of sessile drops showing the water contact angle of unirradiated membranes (a), gamma irradiated with dose of $15 \mathrm{kGy}$ (b), $25 \mathrm{kGy}(\mathrm{c}), 35 \mathrm{kGy}(\mathrm{d})$ and $65 \mathrm{kGy}(\mathrm{e})$.

Table 1: Variation of water contact angle due to gamma irradiation

\begin{tabular}{|l|l|}
\hline Irradiation dose & $\begin{array}{l}\text { Neat PCL } \\
(\text { Degree })\end{array}$ \\
\hline Untreated & $106 \pm 5$ \\
\hline $15 \mathrm{kGy}$ & $94 \pm 0.9$ \\
\hline $25 \mathrm{kGy}$ & $84 \pm 0.6$ \\
\hline $35 \mathrm{kGy}$ & $87 \pm 0.4$ \\
\hline $65 \mathrm{kGy}$ & $79 \pm 2$ \\
\hline
\end{tabular}

electrospun PCL membrane was about $106 \pm 5^{\circ}$, indicating that the water droplet did not spread well on the hydrophobic surface of the membrane. A considerable decrease in water contact angle was observed for all the membranes after the gamma irradiation which indicates an increase in wettability. In the case of membranes irradiated with $35 \mathrm{kGy}$ of gamma, the contact angle decreased to $87 \pm 0.4^{\circ}$ which was about $18 \%$ less than the contact 
angle of unirradiated samples. The highest decrease in contact angle was observed in the case of samples irradiated with $65 \mathrm{kGy}$ which was about $25 \%$ less than the initial value. The contact angle depends on the chemical composition at surface and the solid-liquid interaction. If the interaction between the solid and the liquid is high, the contact angle will be less [18]. Presence of surface polar groups causes a decrease in contact angle due to the higher interaction between the solid surface and the polar solvent [18]. Thus, it is clear that the gamma treatment has significant effects on the formation of polar surface functional groups which increased the hydrophilicity of the membranes.

\subsection{XRD analysis after gamma irradiation}

It is very important to find out how the crystallinity of the polymer changes during the gamma irradiation process since the crystallinity affects mechanical properties of the material. Figure 4 shows the XRD spectra of unirradiated and gamma irradiated PCL

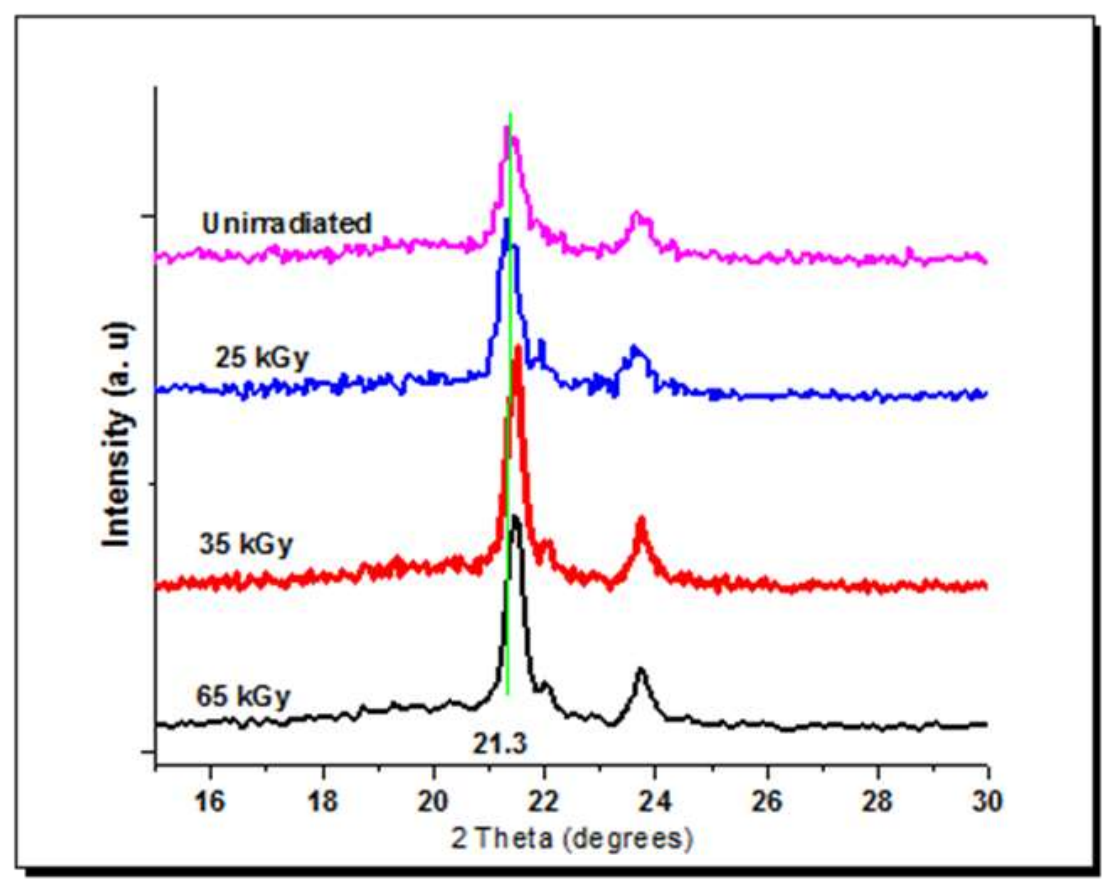

Figure 4: XRD spectra of electrospun PCL membranes irradiated with varying doses of gamma irradiation.

membranes. PCL is a semicrystalline polymer and contains both crystalline and amorphous regions. Results show that electrospun PCL membrane contains three distinct reflections at the Bragg angles of about $21.4^{\circ}, 22.0^{\circ}$ and $23.7^{\circ}$, corresponding to the (110), (111) and (200) planes of the orthorhombic crystal structure respectively [1]. It is interesting to note that the increase in intensity of diffraction peaks at lower doses indicating an increase in the crystallinity of the polymer. This tendency could be observed up to $35 \mathrm{kGy}$ of irradiation 
dose. The overall increase in the diffraction peak intensity suggests the formation of more ordered arrangement of crystallites after gamma irradiation. However, at $65 \mathrm{kGy}$, there was a slight decrease in intensity than that of $35 \mathrm{kGy}$ suggests the disorderness of the crystallites. This decrease in the intensity can be attributed to the decrease in the amount of crystalline phase after irradiating with higher dose of gamma. An increase in irradiation dose does not give rise to new diffraction peaks nor cause the disappearance of existing ones. This means that irradiation-induced scission and crosslinking of macromolecules do not produce macromolecular reorganizations which would be able to create new crystalline symmetries [19].

In order to get the semi quantitative information of the percentage of crystallinity in PCL membranes due to gamma irradiation, from the area of the diffraction peaks and the area of amorphous hallow region, calculated from the XRD software and shown in Table 2.

Table 2: Variation of percentage of crystallinity due to gamma irradiation

\begin{tabular}{|l|l|}
\hline Irradiation dose & Crystallinity (\%) \\
\hline Unirradiated & 64.4 \\
\hline $15 \mathrm{kGy}$ & 66.52 \\
\hline $25 \mathrm{kGy}$ & 67.47 \\
\hline $35 \mathrm{kGy}$ & 69.62 \\
\hline $65 \mathrm{kGy}$ & 65.34 \\
\hline
\end{tabular}

Percentage of crystallinity of unirradiated PCL membrane was $66.4 \%$. The highest percentage of crystallinity among the studied samples was 69.62 which were obtained for sample irradiated with $35 \mathrm{kGy}$. The results show that exposure to lower gamma dose results into the enhancement of crystallinity, which leads to the ordering in the polymer chains [20]. Whereas, in case of membranes irradiated with $65 \mathrm{kGy}$ gamma dose, the percentage of crystallinity decreased and less than the pristine samples.

These results reflect that the amorphous character of the polymer decreases and crystallinity increases with increase in dose, which may be due to the scissioning of the polymer chains, by which the polymer undergoes some spatial rearrangement [21]. The small fragments may rearrange themselves towards new crystalline zone. Whereas the higher dose resulted in the decrease in crystallinity due to the crosslinking of fragmented chains which change the regularly arranged crystallites into non arranged ones by forming new bonds between the neighboring chains. 


\subsection{Tensile testing}

From the tensile measurements, it was observed that as the irradiation dose increases the tensile strength increased as indicated by the increase in break stress and young's modulus which is shown in Figure 5 and Table 3. However, the maximum elongation showed no

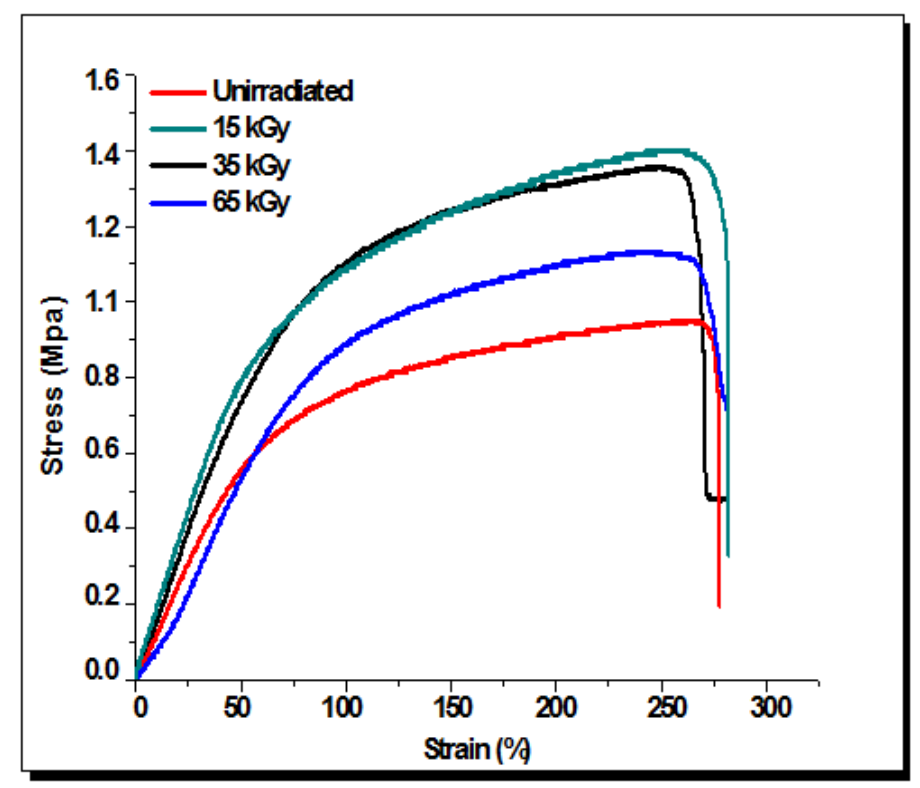

Figure 5: Stress strain curves of electrospun PCL membranes after irradiating with gamma radiations of varying doses.

Table 3. Variation of tensile properties due to gamma irradiation

\begin{tabular}{|l|l|l|l|l|}
\hline Sample & $\begin{array}{l}\text { Gamma irradiation } \\
\text { dose (kGy) }\end{array}$ & $\begin{array}{l}\text { Break stress } \\
(\mathrm{MPa})\end{array}$ & $\begin{array}{l}\text { Maximum } \\
\text { elongation (\%) }\end{array}$ & $\begin{array}{l}\text { Young's } \\
\text { Modulus } \\
\text { (MPa) }\end{array}$ \\
\hline 1 & 0 & 0.98 & 275 & 3.87 \\
\hline 2 & 15 & 1.23 & 280 & 4.13 \\
\hline 3 & 25 & 1.32 & 283 & 4.22 \\
\hline 5 & 35 & 1.18 & 268 & 4.28 \\
\hline
\end{tabular}

significant variation as the radiation dose increases. This variation in the elongation at break and tensile strength can be explained in terms of polymer chain scission and crosslinking. In general, both chain scission and cross linking take place simultaneously. It is plausible to say that the chain scission process is yet to take prominence at these low doses $[22,23]$. The 
fragmented chains undergo crystallization in a large extent than the cross-linked and large macromolecular assemblies. XRD results reflected that the amorphous character of the polymer decreases and crystallinity increases with increase in irradiation dose. This might be due to scissioning of the polymer chains and the possible spatial rearrangement that occurred in the polymer [20]. Highly crystalline polymers show superior tensile properties than amorphous one. The small fragments may rearrange themselves towards new crystalline zone. Whereas the higher dose resulted in the decrease in crystllinity due to the crosslinking of fragmented chains which change the regularly arranged crystallites into non arranged ones by forming new bonds between the neighboring chains. Thus the higher irradiation dose resulted in inferior tensile properties. Maximum elongation achieved by the gamma irradiated membranes, did not show significant differences compared with the unirradiated PCL membranes which is in agreement with the previous studies [20].

\subsection{Effectiveness of sterilization}

If no evidence of microbial growth is found, the product to be examined complies with the test for sterility. If evidence of microbial growth is found, the product to be examined does not comply with the test for sterility, unless it can be clearly demonstrated that the test was invalid for causes unrelated to the product to be examined. This study has shown that the gamma irradiation of electrospun PCL membranes successfully restricted the growth of microorganisms, although the procedure below $35 \mathrm{kGy}$ does not completely eliminate microorganisms from the material. From Figure 6 (a), it is evident that in unirradiated membranes, microorganisms were present and the plates streaked with the thioglycolate medium show growth of microbes. Membranes irradiated with $15 \mathrm{kGy}$ gamma dose also shows apparently similar growth of microbes in the culture plates (Figure 6 (b)). Treatment of samples with $25 \mathrm{kGy}$ gamma irradiation resulted in the removal of some of the microorganisms from the sample surface, although total sterilization was not achieved (Figure 6 (c)). Exposure to $35 \mathrm{kGy}$ of gamma irradiation completely eliminated the presence of microorganisms in the PCL samples (Figure $6(\mathrm{~d})$ ). Although previous reports showed that $25 \mathrm{kGy}$ gamma exposures is enough to eliminate microbial growth [24], our study showed that, at least $35 \mathrm{kGy}$ gamma dosage is required to completely eliminate the microbial growth from the samples (Figure $6(\mathrm{~d})$ ). The more resistant bacteria (spore-forming bacteria) may 


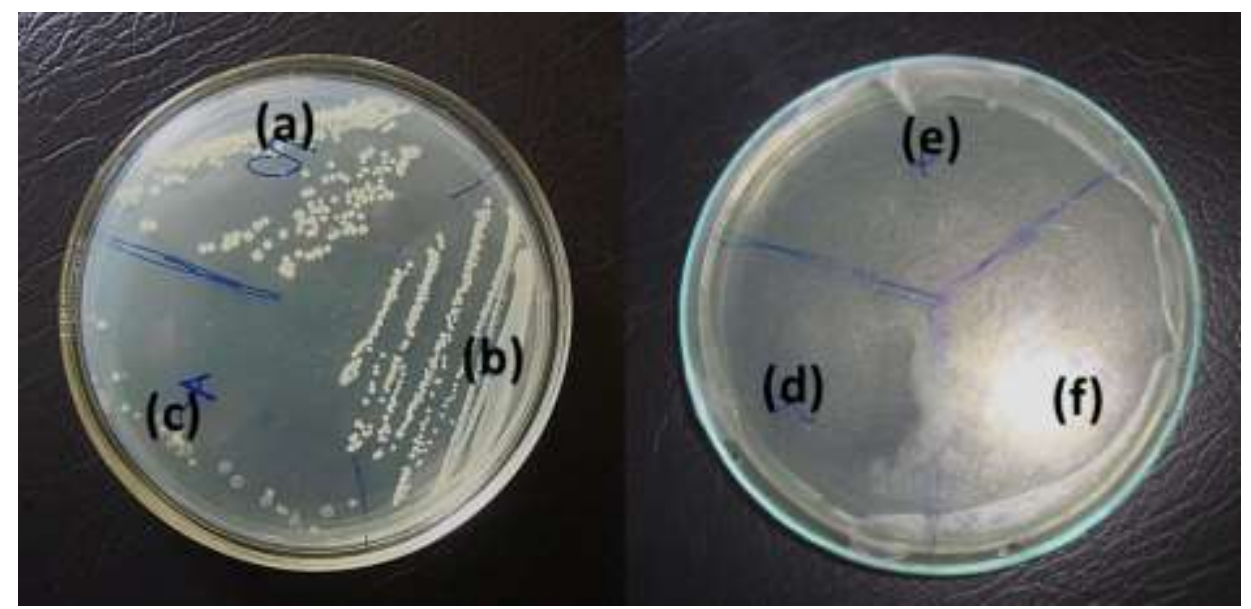

Figure 6: Plate showing the sterility of the gamma irradiated membranes in terms of the decrease in microbial growth after 14 (with sample) +4 (without sample) days of incubation in thioglycollate medium. Streak (a) is unirradiated PCL membrane, (b) is PCL membrane irradiated with $15 \mathrm{kGy}$, (c) is PCL membrane irradiated with $25 \mathrm{kGy}$, (d) is PCL membrane irradiated with $35 \mathrm{kGy}$, (e) is PCL membrane irradiated with $65 \mathrm{kGy}$ gamma radiation and (f) is the control without samples.

survive as high as $30 \mathrm{kGy}$. Application of $65 \mathrm{kGy}$ gamma irradiation also successfully eliminated the presence of microorganisms in the sample (Figure $6(\mathrm{e})$ ).

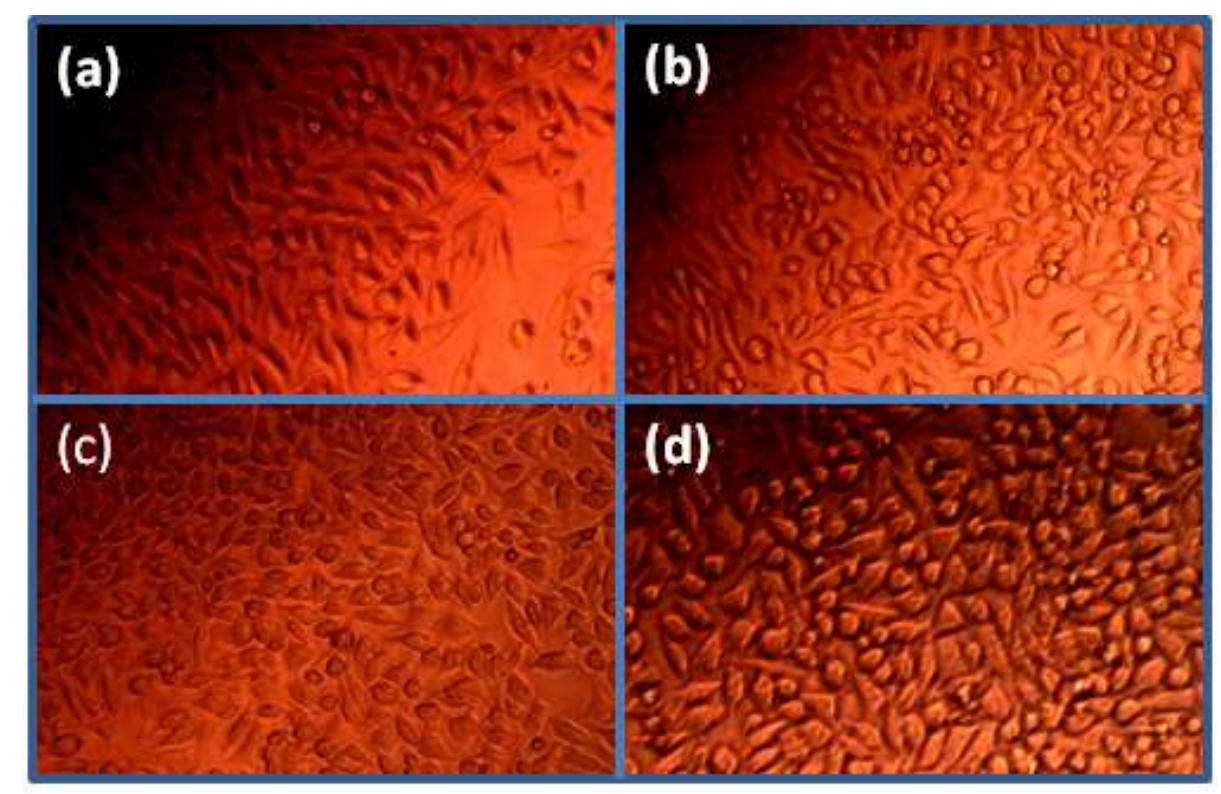

Figure 7: Fibroblast cell proliferation on unirradiated PCL membrane (a), PCL membrane irradiated with 15 kGy (b), 35 kGy (c) and 65 kGy doses of gamma radiation.

\subsection{Fibroblast cell proliferation}

From Figure 7, it is evident that, L929 fibroblast cells grow very well on electrospun PCL membranes that have been irradiated by gamma radiation. This was in agreement with the previous reports [25]. In addition to this, our study had shown that while increasing the 
irradiation dose from $15 \mathrm{kGy}$ to $65 \mathrm{kGy}$, the cell proliferation also increased. It confirmed that gamma irradiation does not adversely affect the proliferation of the fibroblast cells on electrospun PCL membranes but enhances the cell proliferation in dose dependent manner. A previous report also support this observation that polyurethane vascular grafts irradiated with gamma radiation showed superior endothelial cell attachment than the unirradiated counterparts [26].

The mechanism of enhanced cell proliferation on gamma irradiated scaffolds can be explained in terms of the increased hydrophilicity of the irradiated membranes. The results of this study show that the contact angle decreased from $106^{\circ}$ to $79^{\circ}$ during gamma irradiation. Most of the mammalian cells like fibroblasts [27 - 29] were found to have maximum adhesion when the contact angles were between $60^{\circ}$ and $80^{\circ}$. FTIR results showed that while irradiating with gamma radiation, both $-\mathrm{OH}$ and $-\mathrm{COOH}$ groups were formed on the PCL membranes. Polymers with hydroxyl group on the surface showed good cell adhesion, spreading and growth properties [30, 31]. The interaction of human fibroblasts with $\mathrm{COOH}$ functionalized surfaces was also reported and has shown strong attachment, spreading, fibronectin matrix formation and growth [32, 33]. Thus, the increased cell attachment, migration and proliferation on the electrospun PCL membrane might be due to the formation of hydroxyl and carboxyl group on the surface of fibers. A previous work suggested that surfaces with functional groups like $-\mathrm{OH}$ and $-\mathrm{COOH}$ modulate the adsorption of cell adhesion proteins like fibronectin, direct integrin binding and thereby promote cell adhesion [34]. Tamada et al. observed that maximum protein adsorption take place on surfaces with water contact angle ranging from $60^{\circ}$ to $80^{\circ}$ [28]. Another report suggests that surfaces with contact angle between $0^{\circ}$ and $80^{\circ}$ showed greater fibronectin adsorption and more fibroblasts spread with increasing wettability [29]. Thus, it is plausible to suggest that the increased cell proliferation on gamma irradiated PCL membrane was due to the increased hydrophilicity 
due to the formation of $-\mathrm{OH}$ and $-\mathrm{COOH}$ functional groups. This increased hydrophilicity facilitated the adsorption of cell adhesion proteins and thereby the increased cell adhesion and proliferation. A schematic representation of the exposure of surface functional groups and the cell adhesion protein mediated fibroblast attachment on the PCL scaffold is given in Figure 8.

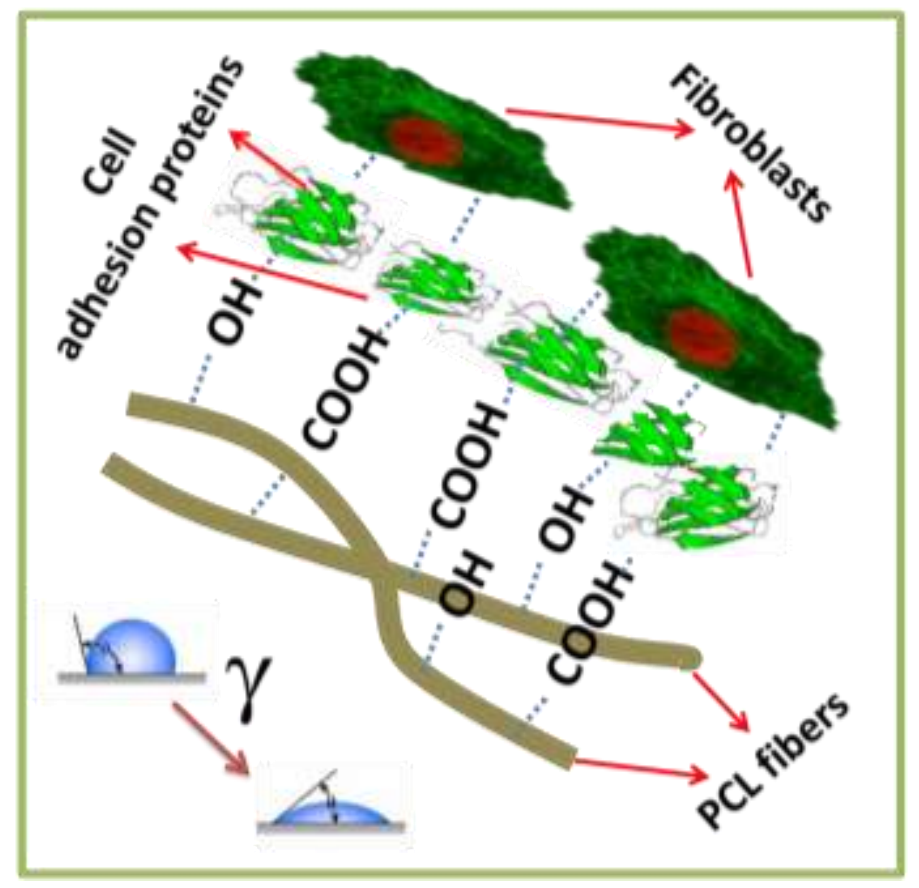

Figure 8: A schematic representation of the exposure of surface functional groups and the cell adhesion protein mediated fibroblast attachment on the gamma irradiated PCL membrane.

\section{Conclusions}

In this work, the sterilizability of electrospun polycaprolactone membranes with gamma irradiation and its effect on the materials property have been investigated. From the SEM analysis, it is clear that there was no considerable variation in fiber morphology during gamma irradiation at any dosage. FTIR analysis clearly indicated the presence $\mathrm{OH}$ (hydroxyl) and $\mathrm{COOH}-$ (carboxyl) functional groups in the PCL membrane after the irradiation due to the cleavage of ester bonds. Water contact angle of all the membranes decreased with increase in irradiation dose which is in agreement with FTIR results. The XRD results show that at lower dose of gamma irradiation, the crystallinity of the membrane increases where as at higher dose, a slight decrease in crystallinity was observed. There was no remarkable variation in the tensile property of the membrane after gamma irradiation, however a slight 
increase at lower dose and slight reduction at higher radiation dose was observed. Application of gamma radiation above $35 \mathrm{kGy}$ completely eliminated the presence of microorganisms in the PCL membranes. Finally, the fibroblast cell growth studies showed that gamma irradiation of electrospun PCL membranes does not inhibit cell attachment and proliferation. Cells were able to grow much better on PCL membranes that have been sterilized by gamma irradiation in a dose dependent manner than the unirradiated ones. Herein from the present study, it is clear that electrospun PCL membranes can be effectively sterilized by irradiating with $35 \mathrm{kGy}$ of gamma exposure which is an optimum dose without compromising the materials properties as well as cell proliferation.

\section{Acknowledgements}

The authors acknowledge Department of Biotechnology (DBT), Government of India, New Delhi for the financial support through MSUB IPLSARE program. Funding from UGCSAP, DST-FIST, DST-Nanomission, SAARD-KSCSTE are also gratefully acknowledged.

\section{References}

1. Augustine, R.; Malik, H. N.; Singhal, D. K.; Mukherjee, A.; Malakar, D.; Kalarikkal, N.; Thomas, S. J. Polym. Res. 2014, 21, 1-17

2. Augustine, R.; Dominic, E. A.; Reju, I.; Kaimal, B.; Kalarikkal, N.; Thomas, S. RSC Adv. 2014, 47, 24777-24785

3. Mirmohammadi, S. A.; Imani, M.; Uyama, H.; Atai, M. Int. J. Polymer. Mater. 2014, 63, 624-631.

4. Augustine, R.; Rajendran, R.; Cvelbar, U.; Mozetič, M.; George, A. Biopolymers for Health, Food, and Cosmetic Applications. Handbook of Biopolymer-Based Materials: From Blends and Composites to Gels and Complex Networks, Wiley-VCH Verlag GmbH \& Co. KGaA, Weinheim, Germany, 801-849.

5. Ng, K. W.; Achuth, H. N.; Moochhala, S.; Lim, T. C.; Hutmacher, D. W. J. Biomater. Sci., Polym. Ed. 2007, 18, 925-938.

6. Zhang, Y.; Ouyang, H.; Lim, C. T.; Ramakrishna, S.; Huang, Z. M. J. Biomed. Mat. Res. Part B: Appl. Biomat. 2005, 72, 156-165.

7. Aghdam, R. M.; Shakhesi, S.; Najarian, S.; Mohammadi, M. M.; Ahmadi Tafti, S. H.; Mirzadeh, H. Int. J. Polymer. Mater. 2014, 63, 229-239. 
8. González, M. E.; Lunati, C.; Floccari, M.; Salmoral, E. M. Int. J. Polymer. Mater. 2009,58, 129-140.

9. Birkinshaw, C.; Buggy, M.; Henn, G. G.; Jones, E. Polym. Degrad. Stab. 1992, 38, 249253.

10. Abadi, F. J. H.; Tehran, M. A.; Zamani, F.; Nematollahi, M.; Mobarakeh, L. G.; NasrEsfahani, M. H. Int. J. Polymer. Mater. 2014, 63, 57-64

11. Aarya, S.; Srivastava, A. K.; Saha, A.; Wahab, M. A. Nucl. Instrum. Methods Phys. Res., Sect. B. 2009, 267, 3545-3548.

12. Jo, S. Y.; Park, J. S.; Gwon, H. J.; Shin, Y. M.; Khil, M. S.; Nho, Y. C.; Lim, Y. M. Radiat. Phys. Chem. 2012, 81, 846-850.

13. Sinha, D. Adv. Appl. Scie. Res. 2012, 3, 1365-1371.

14. Gonzalez, M. E.; Salmoral, E. M.; Traverso, K.; Floccari, M. E. Int. J. Polymer. Mater. 2002, 51, 721-731.

15. Suljovrujić, E.; Ignjatović, N.; Uskoković, D. Radiat. Phys. Chem. 2003, 67, 375-379.

16. Gorna, K.; Gogolewski, S. Polym. Degrad. Stab. 2003, 79, 465-474.

17. Satake, M.; Mido, Y. .; Sethi, M. S.; Iqbal, S. A.; Yasuhira, H.; Tagauchi, S. Environmental Toxicology. Discovery Publishing House. 1997, p. 207.

18. Watson, C. L.; Letey, J. Soil Scie. Society America J. 1970, 34, 841-844.

19. Stojanović, Z.; Kačarević-Popović, Z.; Galović, S.; Miličević, D.; Suljovrujić, E. Polym. Degrad. Stab. 2005, 87, 279-286.

20. Bosworth, L. A.; Gibb, A.; Downes, S. J. Polym. Sci., Part B: Polym. Phys. 2012, 50, $870-876$.

21. Sinha, D.; Sahoo, K. L.; Sinha, U. B.; Swu, T.; Chemseddine, A.; Fink, D. Radiat. Eff. Defects Solids 2004, 159, 587-595.

22. Södergård, A.; Stolt, M. Prog. Polym. Sci. 2002, 27, 1123-1163. 
23. Bovey, F. A. Reactions of Solid Polymers. In Treatise on Solid State Chemistry, Springer US, 1976, pp. 661-714.

24. da Silva Aquino, K. A. Sterilization by gamma irradiation. Gamma irradiation. Croatia: InTech Europe. 2012.

25. Cottam, E.; Hukins, D. W.; Lee, K.; Hewitt, C.; Jenkins, M. J. Med. Eng. Phys. 2009, 31, 221-226.

26. Hsu, S. H.; Chuang, S. C.; Chen, C. H.,; Chen, D. C. Bio-Med. Mater. Eng. 2006, 16, 397404.

27. Altankov, G.; Grinnell, F.; Groth, T. J. Biomed. Mater. Res. 1996, 30, 385-391.

28. Tamada Y; Ikada Y. Polymer 1993, 34, 2208-2212.

29. Wei, J.; Yoshinari, M.; Takemoto, S.; Hattori, M.; Kawada, E.; Liu, B.; Oda, Y. J. Biomed Mat Res Part B: Appl 2007, 81, 66-75.

30. Lee, J. H.; Park, J. W.; Lee, H. B. Biomaterials 1991, 12, 443-448.

31. Lee, J. H.; Jung, H. W.; Kang, I. K., Lee, H. B. Biomaterials 1994, 15, 705-711.

32. Curtis, A. S. G.; Forrester, J. V.; Clark, P. J. Cell Sci. 1986, 86, 9-24.

33. Faucheux, N.; Schweiss, R.; Lützow, K.; Werner, C.; Groth, T. Biomaterials 2004, 25, 2721-2730.

34. Keselowsky, B. G.; Collard, D. M.; García, A. J. J Biomed Mat Res Part A 2003, 66, 247 259. 